\title{
DERMATOMYOSITIS WITH MESENTERIC VEIN THROMBOSIS AND PNEUMOPERITONEUM
}

Adriane Souza da Paz ${ }^{1, \star}$, Anna Paula Duque Motta ${ }^{1}$, Gustavo Luiz Behrens Pinto ${ }^{1}$, Mittermayer Barreto Santiago

1.Universidade Federal da Bahia, Salvador (BA), Brazil.

*Corresponding author: adriane.spaz@hotmail.com

\section{BACKGROUND}

Dermatomyositis (DM) is an autoimmune inflammatory myopathy characterized by symmetrical proximal muscle weakness and skin lesions such as Gottron's papules and heliotrope. It can also involve the gastrointestinal system, but mesenteric thrombosis with intestinal ischemia is rare. We describe a case of DM that evolved with pneumoperitoneum secondary to intestinal perforation due to distal ileum mesenteric venous thrombosis.

\section{CASE REPORT}

A 48-year-old man was diagnosed with DM five years ago with Gottron's papules, muscle weakness and an increase of CPK. He was admitted to our institution with atypical ulcerative skin lesions (Fig. 1), worsening muscle weakness, and severe abdominal pain. Laboratory tests identified hemoglobin $11.0 \mathrm{mg} / \mathrm{dL}(12-16 \mathrm{mg} / \mathrm{dL})$, total white blood counts 16,000 cells/mm3, platelets 120 $\times 109 / \mathrm{L}$, C-reactive protein $73 \mathrm{mg} / \mathrm{dL}$ (<6 mg/dL), CPK 140 (32-211 U/L). During his hospitalization, the abdominal pain became worse, and the investigation with abdominal computed tomography revealed pneumoperitoneum, free fluid in the abdominal cavity, and thickening of the intestinal wall, especially in the ileocecal region (Fig. 2). Exploratory laparotomy identified multiple intestinal perforations and extensive lesion $(60 \mathrm{~cm}$ ) in the ileocecal valve. The histopathological evaluation demonstrated ischemic necrosis and perforations in the small bowel wall and recent mesenteric venous thrombosis (Fig. 3). Despite treatment with corticosteroids and immunoglobulin, he evolved with severe sepsis and death.

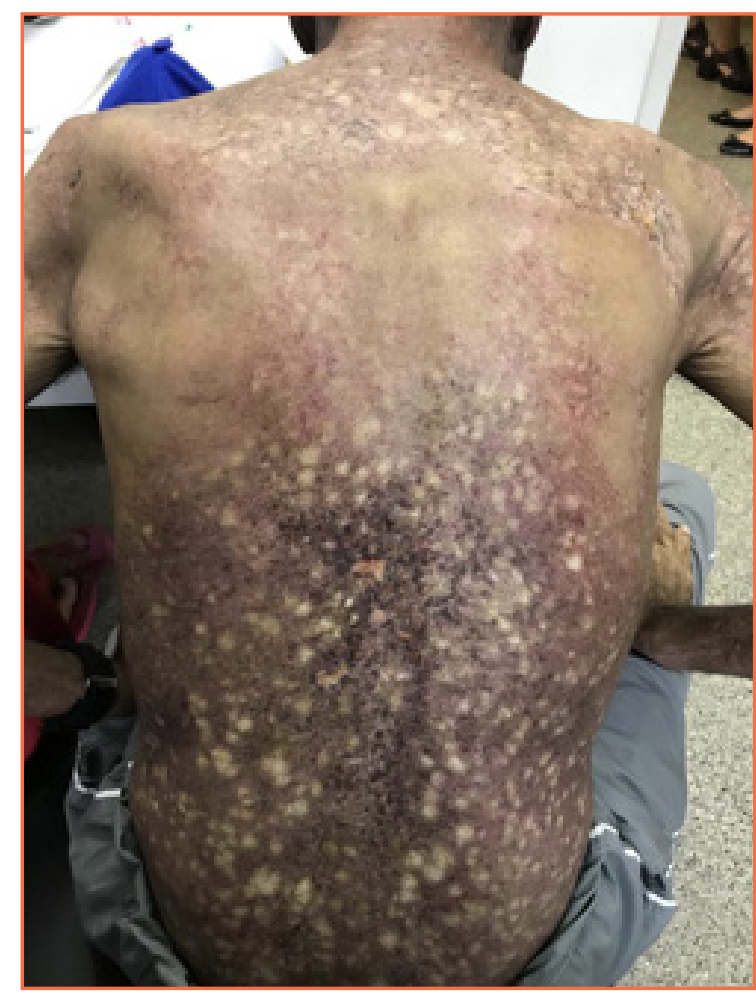

Figure 1. Ulcerative skin lesions and muscle atrophy. 


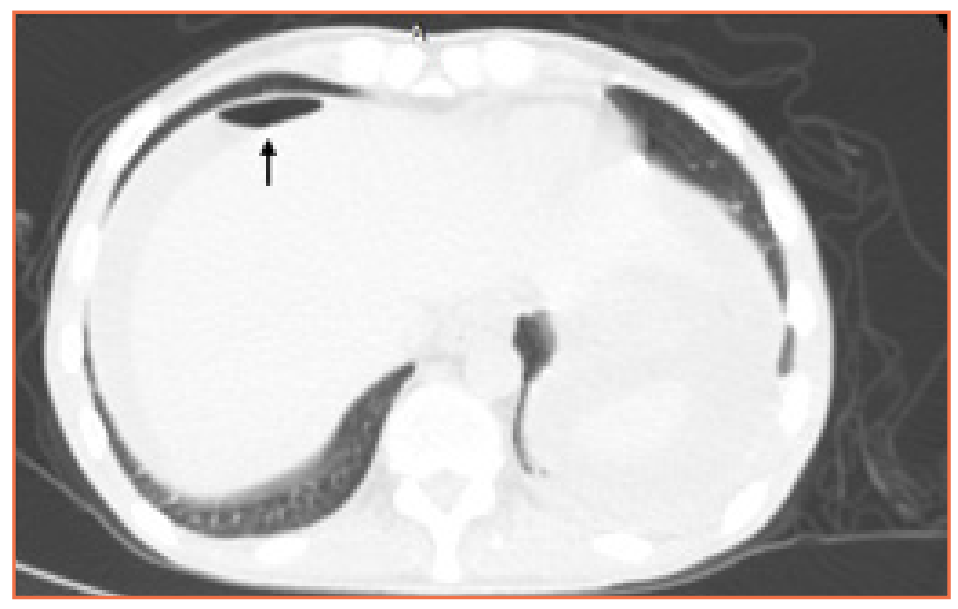

Figure 2. Findings of the abdominal tomography (black arrow: pneumoperitoneum).

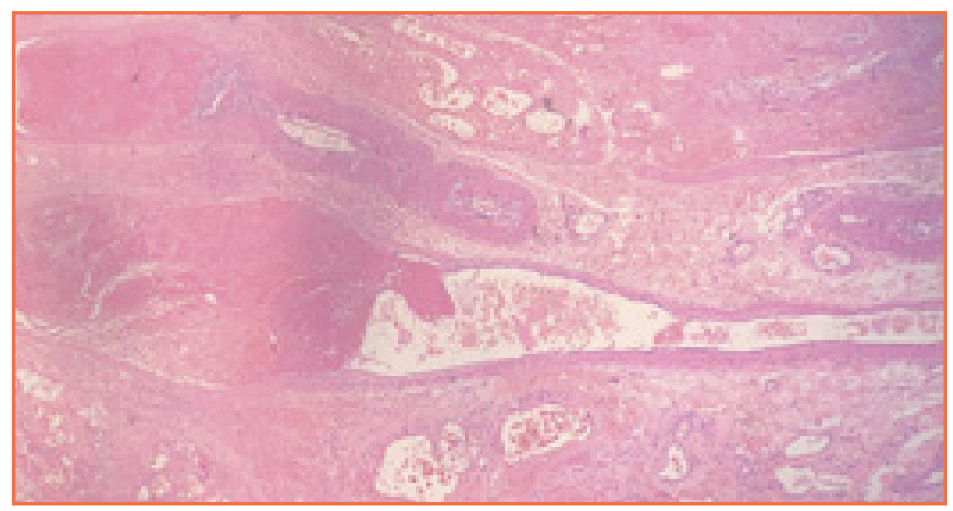

Figure 3. Ischemic necrosis and perforations in the small bowel wall and recent mesenteric venous thrombosis.

\section{CONCLUSION}

Gastrointestinal manifestations occur in $50 \%$ of cases of DM, mainly dysphagia, esophageal and intestinal dysmotility. Intestinal perforation associated to mesenteric venous thrombosis is rare, has an etiopathogenic mechanism unknown, and is associated with high morbidity and mortality. It should be considered in the context of refractory abdominal pain in a patient with DM. 